\title{
Intra and extracranial complications of middle earths: literature review
}

\begin{abstract}
Otitis media $(\mathrm{OM})$ has several forms and among the main are otitis media (AOM), OM with effusion and chronic otitis media (COM) (cholesteatomatous or not). For the most part, the OM progressed well, but there are risks of complications and sequelae. These are divided into extra and intracranial, the first being the most common, but less lethal. Among the extracranial are: labyrinthine fistula, subperiosteal abscess, mastoiditis, temporozigomatic abscess, Bezold's abscess, parapharyngeal abscess, facial palsy, petrositis and labyrinthitis. Among the intracranial are: meningitis, epidural abscess, subdural empyema, brain abscess, the sigmoid sinus thrombophlebitis and otological hydrocephalus. The aim of this study is to identify the main complications of otitis media, distinguishing them from the incidence, degree of morbidity and mortality and analyze the development, management and treatment required for each entity. Brain abscess is the most common entity and subperiosteal abscess is the most common extracranial complication. Labyrinthine fistula occurs most often related to OMC. This has the physiopathology erosion of the bone covering the semicircular canal, usually the lateral semicircular canal. Labyrinthitis results from the spread of infection from the cochlear window membrane and can be presented in two ways: serous or suppurative labyrinthitis. The most common intracranial complication is meningitis, rarely associated with cholesteatoma and most often associated with AOM, of higher incidence in children. CT with contrast is the gold standard when you suspect any complications in patients with otitis media after undergoing a thorough history and physical examination. When the physician knows the possible complications and their signs and symptoms, the diagnosis is early and the prognosis best. Treatment of complications in general is based on patient hospitalization, myringotomy with culture and sensitivity, intravenous antibiotic therapy as early as possible and mastoidectomy in all cases related to COM or recurrent complications.
\end{abstract}

Keywords: otitis media, intracranial complications, extracranial complications

\section{Introduction}

The middle ear is formed by the tympanic membrane (TM), tympanic cavity, mastoid cells, mastoid antrum and auditory tube. It separates from the outer ear by TM and communicates with the inner ear through the vestibular and cochlear windows. Inflammations, infectious or not, from these regions are called otitis media $(\mathrm{OM})$, and represent one of the most prevalent infectious diseases in the world, especially in childhood. This is attributed not only to the fact that the anatomical position of the auditory tube is less favorable (more horizontal, shorter and wider compared to that of the adult), but also because of the inherent immunological immaturity. ${ }^{1}$

There are several forms of presentation of $\mathrm{OM}$ in general and among the main ones are: acute otitis media (AOM) (recurrent or not), OM with effusion and chronic otitis media (cholesteatomatous or not). In the vast majority of cases, OMs have a good evolution, but are considered potentially serious diseases worthy of attention due to their risks of sequelae and complications. The complication, entity that we will address in this study, occurs when the infectious process extends beyond the mucoperiosteum. It is considered a sequel when it is limited to the mucoperiosteum and causes anatomical or functional lesions, even after the resolution of the disease and is not considered a medical emergency. The form of the disease that the patient will develop depends on several factors, such as age, local and systemic immunity, virulence of the causative agent and previous treatments. ${ }^{2}$

The middle ear has an important relation with noble structures. Superiorly it is limited with the middle cranial fossa, through a thin bony blade. Later, with the posterior cranial fossa and sigmoid
Volume II Issue 2 - 2019

\author{
Carlos Eduardo Borges Rezende, Stephanie \\ Rissio, William José Gilioti, Morgana Moreno \\ Boschi \\ Otolaryngologist, Brazi
}

Correspondence: Carlos Eduardo Borges Rezende, Otolaryngologist, CRM 94665, Av Pereira Barreto I 395 Cj 34, North Tower Paradise Sto André, Brazil, Tel 4425 0485, 96403 4192, Email cadurezendel0@gmail.com

Received: June 20, 2017 | Published: April 02, 2019 sinus. Inferiorly the hypotympanum is separated from the sigmoid sinus and the jugular bulb by another thin bony layer. Medially, the cochlear window and the stapes platinum are its limits. These intimate relationships contribute to infections in this region having a high potential for complications. ${ }^{1}$

These complications may spread through bone erosions secondary to infections, through thrombophlebitis, previously existing pathways (cochlear window, congenital bone dehiscence) or non-anatomical bone defects (trauma, neoplastic erosions). ${ }^{3}$ They are divided into extracranial and intracranial, the first being the most common, but less lethal. Among the extracranial ones are: labyrinthine fistula, subperiosteal abscess, mastoiditis, temporozygous abscess, Bezold's abscess, parapharyngeal abscess, peripheral facial paralysis, petrositis and labyrinthitis. Among the intracranial ones are: meningitis, epidural abscess, subdural empyema, cerebral abscess, thrombophlebitis of the sigmoid sinus and otogenic hydrocephalus. ${ }^{4,5}$

The incidence and morbidity and mortality of complications from OM decreased dramatically after the advent of the antibiotic, more precisely after the fifth decade of the last century. However, its indiscriminate use may mask the symptoms of a more advanced disease and delay the diagnosis, in addition to favoring bacterial resistance. The physician should always be aware of symptoms such as: otorrhea, elevated fever, headache, otalgia, hearing loss, vertigo, nausea, vomiting, suppuration, signs of peripheral facial paralysis (PFP), dysphagia, nuchal rigidity, among others. ${ }^{6}$ In the pre-antibiotic era, intracranial complications secondary to otological diseases occurred in $2.3-6.4 \%$ of cases, and the mortality rate was approximately $75 \%$. In the post-antibiotic era, the incidence decreased 
to $0.04-0.15 \%$ of cases. Mortality also showed a significant reduction, but is still relevant. Currently, with the routine use of antibiotics for the treatment of otitis media, improvements in temporal bone-related surgical techniques and more effective imaging, there is a better prognosis for complications in general, since the diagnosis occurs earlier and the treatment is more. ${ }^{7}$ One of the main risk factors for the development of intra-or extracranial complications is the difficulty of access to the public health system, the precariousness of care and the lack of education of the population, as evidenced by the lower rates in developed countries.

\section{Goals}

To distinguish intra and extracranial complications of otitis media in terms of incidence, morbidity and mortality, and to comprehensively analyze the evolution, behaviors and treatments necessary for each entity.

\section{Methodology}

The present study consists of a review of the scientific literature and includes material from the last 15 years. The articles identified by the initial search strategy were selected according to the following inclusion criteria: (1) population: adult and pediatric. (2) Etiology: complications of otitis media. (3) Clinical manifestations of intra and extracranial complications. (4) Incidence of complications (5) approach in the diagnosis and treatment of each complication. The descriptors used were: (1) otitis media, (2) complications, (3) intracranial, (4) extracranial. The selection resulted in 24 articles in the following languages: English and Portuguese, from 2002 to 2014.

\section{Literature revision}

\section{Etiologic agents of complications}

It is extremely important to know the main microorganisms involved in intra and extracranial complications, as this will guide the physician to empirically choose the most effective antibiotic for each case, and to respond to the best results of clinical treatments, sometimes avoiding more invasive measures. ${ }^{3}$ In cases of extracranial complications related to AOM, the most commonly encountered agents are: Streptococcus pneumoniae, Haemophilus influenzae and Moraxella catharralis. In the intracranial complications by AOM, the same agents exist, but Haemophilus influenzae type B is found more frequently, especially in cases of meningitis. In a study that reviewed 92 children with OM-associated meningitis, $68 \%$ of cultures were found to be positive for such agent. Other studies show the incidence of $39 \%$ and $20 \%$ of cases of otogenic meningitis with positive culture for the same..$^{8,9}$

In the extracranial complications associated with the WTO, Staphylococcus aureus and Pseudomonas aeruginosa are the main agents. In the intracranial ones we already have Proteus, Streptococcus, anaerobic and gram-negative. In the cases of cholesteatomatous OMC (OMCc) the flora found is usually multibacterial, being Pseudomonas aeruginosa, Proteus spp, Staphylococcus aureus and Bacteroides spp the most frequent agents. ${ }^{1,10}$

\section{Extracranial complications}

More common than intracranial complications, extracranial complications originate mainly from the $\mathrm{OMC}$, and most of them are from OMC. ${ }^{5}$ The subperiosteal abscess is the most frequent form of complications from middle ear pathologies. However, in cholesteatomatous diseases, labyrinthine fistula is the most prevalent entity. ${ }^{7}$ There is a slight predominance in males ${ }^{11,10}$ the mean age ranges from 30 to 50years, and in children it is more common that the complication originates from AOM. In adults and older children, the complications are from the OMC, especially cholesteatomatous, due to the destructive nature of the disease. ${ }^{5,6}$ Unlike intracranial complications, extracranial complications are treated exclusively by the otorhinolaryngologist, be it clinical or surgical intervention. Among the main surgical treatments are mastoidectomies, myringotomy for culture and/or tube placement for ventilation, associated with appropriate antibiotic therapy. ${ }^{8}$

\section{Subperiosteal abscess}

It is the most common extracranial complication. ${ }^{1,12}$ Occurs after perforation of the mastoid cortex. More frequent in children, due to the smaller thickness and greater bone fragility of the mastoid. It is estimated that $4 \%$ of the AOM and $1 \%$ of the WTO develop with this type of complication. There is retroauricular bulging, antero-inferior displacement of the auricle and deletion of the retroauricular groove. The patient has fever, severe pain, toxemia and sometimes otorrhea. The choice of diagnosis for the diagnosis is computed tomography (CT), which evidences bone erosion, loss of mastoid septations (mastoiditis coalescente) and subperiosteal collection. ${ }^{1,9}$ Treatment is based on intravenous antibiotic therapy and drainage of the abscess. In cases of OMC, perform mastoidectomy. ${ }^{1}$

\section{Labyrinthic fistula}

The most common extracranial complication associated with cholesteatoma is the erosion of the bone that covers the semicircular canal, usually the lateral semicircular canal. ${ }^{1,6}$ In this paper, However, it may be accompanied by fistulas in the posterior, superior and more rarely semicircular canal in the cochlea. ${ }^{13}$ Clinic may range from absence of symptoms to sensorineural hypoacusis, tinnitus, otorrhea, vertigo, nystagmus, nausea and vomiting. ${ }^{13}$

When the fistula is of the lateral semicircular canal, the nystagmus is always horizontal. When it is superior or posterior, nystagmus is vertical. In cases of anterior fistula and ampulla, lateral and superior semicircular canals, nystagmus is rotatory. The main diagnostic indicator is the positive fistula test; in this test, nystagmus and vertigo occur when there is an increase in pressure in the external acoustic meatus. A positive test produces nystagmus with rapid component ipsilateral to the affected side. The negative test does not rule out the diagnosis. ${ }^{13}$ There are specific tests to trigger nystagmus in patients with fistula or semicircular canal dehiscence. There is the Hennebert test, in which pressure is exerted on the tragus and this can be transmitted to the tympanic membrane and ossicular chain, triggering nystagmus. It is the Túlio test, in which intense noises cause nystagmus by a mechanism similar to that of tragus compression. ${ }^{14}$

Often their diagnosis is made only intraoperatively, needing the doctor to be aware of the symptoms to suspect this complication. The high resolution tomography is the most used. It can diagnose cholesteatoma, involvement of the ossicular chain (the long anvil process being the most affected region), labyrinthine fistula and defect in the facial nerve canal, but the sensitivity of this examination varies according to the DORNELLES et al ${ }^{15}$ The treatment is surgical and controversial. Some authors recommend removing all diseased tissue (cholesteatoma) and covering the fistula with fascia of the temporal, 
perichondrium, vein or muscle. ${ }^{11}$ Others, in addition to this option, report on the method of maintaining the cholesteatoma matrix and removing the rest of the tissue. The site of the fistula and the extent of the fistula must be taken into account, due to the risk of lesions of membranous structures of the labyrinth. ${ }^{1,9}$ With regard to preoperative audiometries, the patients present, in general, with mixed hearing loss. This loss is not completely reversed in the postoperative period, but there is improvement in most cases. ${ }^{5}$

\section{Mastoiditis}

The anatomy of the temporal bone is connected to the middle ear by the adductus and the antrum, so all patients with middle ear disease have some degree of inflammation of the mastoid. ${ }^{1}$ When the process progresses, there is lysis of the mastoid bone septations, a process that occurs mainly in $\mathrm{CBC}$ and in patients with years of disease duration. Due to the anatomy cited above, by some authors, mastoiditis is not considered a complication but a natural evolution of otitis media. ${ }^{9}$ In the pre-antibiotic era mastoiditis was very common, more than $20 \%$ of cases of AOM evolved to such a complication. But after the advent of the antibiotic, that rate dropped to less than $2 \%$. In recent years, there has been a resurgence of the disease, which may be associated with bacterial resistance due to the indiscriminate use of the drug. ${ }^{16}$

First there is hyperemia, as the first reaction of mucoperioste to bacteria. This process begins in the auditory tube and goes to the antrum and the cells of the mastoid. There is a change in ciliary function, with damage to middle ear drainage. Next, the exudation phase occurs, in which the serous contents may become purulent. If the process is not resolved, the osteoclasts and vascular neoformation remove decalcified portions, forming the coalescent mastoidite. Normally, coalescent mastoiditis forms two weeks after the onset of otorrhea. ${ }^{1}$ The signs and symptoms often found are: otalgia, otorrhea, tinnitus, retroauricular pain, erythema, edema, pain in the mastoid process and bulging in the bone meatus adjacent to the antrum. Diagnosis is made through clinical and computed tomography, which can demonstrate areas of bone erosion, with loss of septations and hydroaeal levels in the mastoid cells. ${ }^{1}$

The treatment of mastoiditis consists of hospitalization, intravenous antibiotic therapy, with activity on the most frequent etiological agents and myringotomy, associated or not with the placement of transthympanic ventilation tubes. In situations of suspected complications or in the absence of improvement at 48 hours of antibiotic therapy, CT of the temporal bones and eventual mastoidectomy should be performed. In cases of coalescent mastoiditis, mastoidectomy should always be performed, since the risk of intracranial complications is high. ${ }^{1,16}$

\section{Abscess of bezold}

It is a rare complication, due to the cortical rupture of the mastoid tip. There is dissection of the abscess from the tip of the mastoid to the cervical region, posteriorly to the sternocleidomastoid muscle, between the tip and the digastric muscle. ${ }^{1}$ The clinical picture is characterized by pain in the mastoid region, torticollis at clinical examination and increase in the region of the superior insertion of the sternocleidomastoid, causing the retromandibular depression to go out. It may complicate with anterior mediastinitis, which is serious. ${ }^{17}$ The diagnosis should be based on anamnesis and physical examination and, often, the duration of symptoms ends up being a more important factor than the severity of the clinical picture. ${ }^{17}$ The patient may sometimes present few symptoms and give the false idea of being a non-serious condition. Even if the diagnosis is clinical, computed tomography with contrast is indispensable for diagnostic confirmation and surgical planning. It requires drainage of the abscess, intravenous antibiotic therapy and mastoidectomy with cervical exploration. ${ }^{1}$

\section{Facial paralysis}

Peripheral facial palsy (PFP) may be due to both an AOM and an evolution of the OMC. The facial nerve is usually compromised in its tympanic portion and may be involved by several mechanisms such as: bone erosions of the facial canal associated with cholesteatoma or granulation tissue, congenital facial nerve canal dehiscences, spread of infection from small caliber veins. Which drain the middle ear mucosa and lead to toxic neuritis or compressive edema. Channel dehiscences allow inflammatory mediators to injure the nerve. Even in cases of compression, the dysfunction is only observed after $50 \%$ of the nerve fibers have been compromised. ${ }^{1,5}$

PFP secondary to OM usually presents unilaterally, more commonly incomplete, associated with otologic symptoms. It presents, in most cases, suddenly, being rare it is progressive..$^{1}$ It can be presented from Grade I of House-Brackman to grade VI, and patients with higher degree of paralysis usually present cholesteatoma. ${ }^{5}$ The PFP associated with AOM is more common in children, due to the higher incidence of this disease in this age group. However, adults are 10times more likely to develop PFP as a complication. ${ }^{18}$ The prognosis is generally good and should be based on electrical tests with Hilger stimulator or electroneurography. The topography should be accessed through the lacrimation study, the stapedial reflex and imaging tests. The image also helps in the planning of surgical cases. ${ }^{18}$

Treatment varies according to its etiology. In cases of PMA associated with AOM, it is treated with broad-spectrum antibiotic therapy, associated with anti-inflammatory corticosteroids. Myringotomy with secretion culture, with or without placement of the tube for ventilation is also indicated. Mastoidectomy with or without decompression of the facial nerve is indicated only in cases where patients have exacerbation of symptoms of AOM or PFP even with treatment. In addition, in cases where PF grade VI remains after 3 weeks of clinical treatment ${ }^{5}$ or have motor degeneration greater than $90 \%$ in electroneurography. Usually the diagnosis is worse when related to the WTO. ${ }^{1}$

In cases secondary to the WTO, surgery is mandatory and should be performed as soon as possible. The choice between open or closed tympanomastoidectomy occurs from intraoperative findings. ${ }^{1}$ And some authors point out that manipulation of the nerve sheath should be avoided. ${ }^{1,9}$

\section{Labirintite}

In cases of labyrinthitis, the spread of infection occurs from the membrane of the cochlear window. It can be presented in two ways: serous or suppurative labyrinthitis. ${ }^{1,9}$ Serous labyrinthitis occurs by the spread of the toxic products of the bacteria or the inflammatory toxins to the inner ear. The inflammation of the labyrinth is sterile. The symptoms are: dizziness, sensorineural hearing loss, tinnitus and less frequently, nystagmus. In this clinical form, the patient usually recovers, but there may be residual hearing loss. ${ }^{1,9}$ In suppurative labyrinthitis, the bacteria are disseminated, causing more important lesions in the vestibular and auditory system. The invasion occurs via meninges and is usually bilateral or unilateral. When unilateral, 
the infection occurs via the cochlear window, secondary to middle ear disease. Its clinical picture is: intense dizziness, incapacitating, associated with nausea and vomiting, with profound hearing loss, uni or bilateral and tinnitus. Nystagmus is an important sign in the suppurative form and has its rapid component towards the affected ear. However, in processes that are already more evolved, this component is in the opposite direction.

In any form of labyrinthitis, computed tomography should be performed to exclude other complications, ${ }^{1}$ be they extra or intracranial. Magnetic resonance imaging (MRI) in T2 and T1 with contrast can show changes from the early (inflammatory) phases, which computed tomography is not capable of the phases consist of an acute stage with purulent secretion in the perilymph and endolymph, with posterior fibrosis and ossification. ${ }^{1}$ The antibiotics used in these cases should overcome the blood-brain barrier, in addition to controlling the vertiginous crisis with antiemetics and antivertiginosos. ${ }^{1}$ Patients are treated with fourth generation antibiotics. In patients with suppurative labyrinthitis, surgical drainage of the labyrinth may be necessary if conservative therapy fails. If CT shows bone erosion, it is necessary to adopt the labyrinthine fistula. ${ }^{8}$

Myringotomy should be performed for culture and drainage. Surgery is indicated only in refractory cases. ${ }^{1,9}$ Cawthorne and Cooksey exercises can assist in vestibular rehabilitation and are characterized by a rehabilitation program involving head, neck and eye movements, postural control exercises in various positions (sitting, bipodal and unipodal support, walking), use Of soft support surface for decreased proprioceptive input and closed-eye exercises to abolish vision. These exercises implement subsidies for new rearrangements of peripheral sensory information to occur, allowing new patterns of vestibular stimulation, necessary for new experiences, to be performed automatically. ${ }^{19}$

\section{Petrosite}

The infection can spread from the middle ear to the cells of the petrous apex, characterizing petrositis. This may be posterior or perilabirinic and anterior. ${ }^{1}$ They are dangerous infections due to their proximity to the middle and posterior cranial fossa, with a high risk of intracranial complications. ${ }^{7}$ Its symptomatology is related to neuralgic pain, due to its proximity to the trigeminal ganglion and the VI pair. Pain in the deep retro-orbital region is the most common symptom. ${ }^{1,9}$ Petrositis may present with Gradenigo's Syndrome, described by Giuseppe Gradenigo in 1905, which presents the following triad: otitis media (usually otorrhea), retrocorbic pain and paralysis of the IV pair (abducent nerve), which generates diplopia. ${ }^{20}$

The suspicion of this complication should be raised when a patient with $\mathrm{BM}$ has severe pain at the auricular and mastoid topography, with trigeminal irradiation. ${ }^{1}$ For diagnostic confirmation, order computed tomography or magnetic resonance imaging (MRI). The treatment consists of parenteral antibiotic therapy and surgical drainage of perilabirintic cells and the petrous apex. Clinical treatment should be maintained for at least one month after clinical improvement. ${ }^{1}$

\section{Intracranial complications}

Although they are less frequent than the extracranial complications, they deserve attention due to the high mortality rate still found: up to $36 \% .{ }^{1,4}$ This rate varies according to the socioeconomic conditions of each location. In Brazil, for example, the mortality rate varies from 0 to $9 \%$, with morbidity of $24 \% .{ }^{1}$ In the pre-antibiotic era, these rates were much higher. The incidence of intracranial complications secondary to otitis media decreased from $2.3 \%$ to $0.04 \%$. However, people who depend on the public health system and face large waiting times for surgeries have higher rates. Perhaps the greatest risk factor for the development of these diseases is the delay in the intervention, resulting in a longer evolution of the disease. ${ }^{4}$ The most common intracranial complication is meningitis and the most lethal cerebral abscess. Associations of intracranial complications are common. They may occur at any age, although the first to third decade is more prevalent. It is frequently associated with the OMC, mainly cholesteatomatous; although meningitis is closely related to AOM. ${ }^{21}$

The dissemination pathway is, in most cases, through bone dehiscences in the tegmen tympani or the antrum, bone dehiscence in the cavum tympani, vascular channels directly to the lateral sinus, upper petrosal sinus, vascular anastomoses, carotid-tympanic canaliculi, plexus Venous pericardium, cavernous sinus, endolymphatic sac, fistula of the optic capsule or of osteitis in the sinodural angle or petrous apex, as well as empyema of the cochlear aqueduct or perineural spaces in the internal acoustic meatus. Every doctor should maintain a high degree of suspicion to ask for the necessary tests and make the diagnosis early. Persistent headache, fever, otalgia, purulent otorrhea, nausea and vomiting, lethargy, neck stiffness, diplopia, hemianopsia, papilledema, loss of visual acuity, ataxia, convulsion, tremor of intention, aphasia, Dysmetria and dysdiadochokinesia. It is worth noting that in patients undergoing antibiotics, the symptoms may be masked and poor, making diagnosis difficult. ${ }^{22}$

The most appropriate way to avoid intracranial complications is early surgery and the correct use of antibiotics. To assist, computed tomography (sensitivity up to $87 \%$ ) is requested, which, in addition to confirming the diagnosis, complements the surgical planning. ${ }^{23}$ Unlike extracranial complications, intracranial complications are not treated exclusively by otorhinolaryngologists, often requiring the intervention of a neurosurgeon..$^{24}$

\section{Meningitis}

Meningitis is the most common intracranial complication, rarely associated with cholesteatoma and frequently associated with AOM. Other intracranial complications associated with meningitis are common. The spread of the meningeal infection occurs by direct invasion of the disease from inflammations near the meningeal, such as abscess and thrombophlebitis or by bone dehiscences. The hematogenic pathway is also involved in the pathophysiology of the disease, from the infected ear, which occurs more frequently in AOM. It affects children more often. There was a decrease in its mortality rate, however, in Brazil, it still rotates around 20 to $30 \%$, with even greater morbidity. ${ }^{1}$

Signs and symptoms include: severe holocranial headache, fever, nausea and vomiting, photophobia, lethargy, stiff neck, Kernig and Brudzinski signs present. Such signs and symptoms present a rapid onset, which should be sufficient for the physician to suspect meningitis. In neonates, bulging fontanelles and cervical hypotonia may accompany more nonspecific symptoms.

If meningitis is suspected, CT should be ordered to rule out the chance of tonsillar herniation by doing the puncture and then lumbar puncture. CSF is associated with pleocytosis, protein concentration, and low glucose levels. The presence of bacteria in the cerebrospinal fluid confirms the diagnosis. However, when related to cholesteatoma, they may be aseptic. 
The treatment is intravenous antibiotic therapy as early as possible. If related to OMA, it must have spectrum for Gram-positive (always remembering Haemophilus influenzae type B, Gram negative). Already, if related to the WTO, spectrum for Gram-negative and anaerobic. In addition, perform myringotomy with culture and antibiogram. In cases of WTO-related meningitis or recurrent meningitis, mastoidectomy is indicated for the control of adjacent disease and exploration of the middle ear to seek communication with the central nervous system.

\section{Sigmoid sinus thrombophlebitis}

The inflammation of the venous sinus (phlebitis) with the formation of an intramural thrombus (thrombophlebitis) secondary to diseases of the middle ear is due to the anatomical relationship of the venous sinus to the middle ear. The thrombus may partially or totally occlude the sinus, with the possibility of releasing septic emboli. It occurs more frequently in adults, due to its greater relation with the WTO. Although it is a serious complication, it is still underdiagnosed due to nonspecific symptoms and frequent relation with other complications, with symptoms predominating. Among the risk factors, the mastoid, when it is poorly pneumatized, may contribute to local infection. Another known granulation tissue factor is relevant in the development of thrombophlebitis. In one study granulation tissues were found in more than $60 \%$ of the patients.

The clinical picture may be non-specific and oligosymptomatic. Signs and symptoms include headache, lethargy, irritability, fever, chills, otalgia, bulging and pain on retromastoid palpation, papilledema, and other symptoms of concomitant complications. If there is extension to the jugular vein, the patient will present torticollis and bulging of the anterior border of the sternocleidomastoid muscle. Contrast CT may suggest the diagnosis. The "Delta Signal" (caused by the inflammation of the soft tissues surrounding the dura mater that lines the lateral sinus, impregnating itself with the contrast) is characteristic. Angiography, venous phase, MRI and digital subtraction angiography are the most sensitive tests for diagnosis. MRI can delineate the extent of the lesion, the involvement of surrounding structures, and assess blood flow. Angioresonance is also a good examination because it is not invasive and has good sensitivity.

Treatment is controversial. It is increasingly the line of a treatment with greater conservatism. Something in common is the approach with intravenous antibiotic therapy with good passage to the CNS and mastoidectomy to remove all diseased tissue, including granulation tissue, with sigmoid sinus exposure. The operation with thrombectomy and ligation of the jugular vein should be restricted to cases in which there is septic embolism. However, ligation of the jugular vein may not prevent the septic plunger, since collateral circulation is present. Its ligature offers risk of injury to the vagus, accessory and hypoglossal nerve and should be done in restricted cases.

Another discussion of the literature is the use or not of anticoagulants. The tendency is not to use them, since the sigmoid sinus usually recanalizes after weeks, even without the use and risk of haemorrhage and dissemination of the thrombus is avoided. Removal of diseased tissue in mastoidectomy causes the sinus to recanalize spontaneously in most cases.

\section{Epidural abscess}

The most common among intracranial abscesses, is found mainly in the temporal or posterior region and is mostly secondary to OMCc, as well as coalescent mastoiditis. It is installed between the skull and the dura, in a virtual space, so the correct term would be abscess, not empyema. It has an insidious and non-specific clinical picture, making diagnosis difficult. It is often an intraoperative finding or imaging tests. The symptomatology is basically persistent headache, otalgia and thick, pulsatile otorrhea. The patient may present with fever and malaise. In rare cases there may be meningeal irritation (dura mater), worsening of the condition and mild stiffness of the neck. If the jugular vein is compressed, the otorrhea exacerbates.

CT reveals areas of limited extra-axial hypodensity, with thick and irregular contrast uptake at the periphery. However, it is not a good exam for the early stages of the disease, with RM being better. The treatment consists of treating the otologic disease, with mastoidectomy and drainage from the purulent collection. In addition, intravenous antibiotic therapy.

\section{Subdural empyema}

Subdural empyema is less frequent than epidural abscess, but more severe and lethal. The purulent collection installs between the dura mater and the arachnoid, pre-existing space, so the term empyema is correct. It is a neurosurgical emergency. Signs and symptoms, initially, are nonspecific: severe headache, fever, and toxemia. As the collection increases, focal neurologic signs such as hemiplegia, oculomotor deficit, and seizures may occur, indicating its location. Other signs associated with intracranial hypertension, such as vomiting, papilloedema, and altered level of consciousness may be present.

They are poorly related to AOM and more associated with frontal rhinosinusitis. All patients with otitis media and rapid neurological deterioration, seizures and hemiplegia should be investigated for this complication. It usually occurs by direct extension or by thrombophlebitis, which is less common. Your diagnosis is made by CT. An extracerebral hypodensity zone will be found in contact with the skull cap, surrounded by a hyperintense zone (after contrast), which corresponds to reactional arachnoiditis. It should then be drained by a neurosurgeon, through a craniotomy, as soon as possible, followed by exploration of the otological focus. The treatment also consists of broad-spectrum antibiotic therapy, intravenous, in high doses. It presents risk of neurological sequelae and high mortality.

\section{Intracerebral abscess}

It is the most lethal complication of otitis media, it occurs more frequently in adults with $\mathrm{OMC}$, and may be accompanied by meningitis in some cases. The most frequent location is the temporal lobe, followed by the cerebellar region, and the latter is even more aggressive. Mortality is associated with the neurological condition in which the patient is admitted. It is the progression of an extradural abscess, which progressed to subdural and reached the parenchyma. The signs and symptoms are also non-specific and vary by location. They present three clinical stages: encephalitis, latent phase and clinical stage. Encephalitis has inflammation and encephalic edema. At this stage, the symptoms are headache, nausea and vomiting, apathy, irritability, high fever, altered level of consciousness and mild nuchal rigidity, which can be mistaken for a viral condition. CSF has increased protein, with normal cellularity and glucose. The second stage is the latest phase, oligosymptomatic, with low fever, malaise, decreased appetite, lethargy, irritability, which may be asymptomatic. It varies from days to months. CSF does not show changes. In the third 
clinical stage, there is an abscess already formed in the initial area of the inflammation. Constant headache, jet vomiting, bradycardia, extraocular muscle paralysis and papilledema represent signs and symptoms due to intracranial hypertension and/or compression of a specific area. With adequate treatment, it can evolve to cure after the first phase. When the involvement is cerebellar, signs and symptoms such as occipital headache, nystagmus, dysdiadochokinesia and incoordination of the ipsilateral musculature may be present.

CT and MRI make the diagnosis. In the TC, a hypodense, homogeneous, circular zone, causing the compression of neighboring structures, surrounded by a hyperintense contour, is reinforced after contrast, associated with a halo of cerebral edema. The abscess capsule can be easily seen when the case is chronic. MRI with gadolinium is more sensitive and allows for an earlier diagnosis. Both tests can be used as a guide in surgery. The management involves fourth generation antibiotic therapy, intravenous, associated to the surgical procedure, composed of the drainage from the craniotomy, done by a neurosurgeon. When associated with the OMC, mastoidectomy should be performed to explore the otologic focus. It is defined whether it will be done in the same surgical time or not, depending on the patient's condition. Radiological follow-up is necessary. There may be neurological sequelae.

\section{Otitic hydrocephalus}

Considered as a pseudotumor, it is an increase in intracranial pressure above $300 \mathrm{mmH}_{2} \mathrm{O}$, without abscess or meningitis and with normal CSF. The etiology is not well established, but it seems to be associated with sigmoid sinus flow obstruction with extension to the sagittal sinus, which would lead to an alteration of the CSF absorption by arachnoid granulations. The patient has signs and symptoms of intracranial hypertension, such as headache, lethargy, nausea and vomiting, and papillary edema. In addition, it may have diplopia due to paralysis of the VI pair. The diagnosis is exclusion. MRI is required to assess for ventricular dilatation. The lumbar puncture confirms the suspicion, in which there is normal cellular fluidity and high pressure. Intracranial hypertension is controlled with systemic corticosteroids, mannitol, diuretics, and water restriction. Repeated lumbar punctures may also be performed when pressure cannot be controlled with other measures, although there is a risk of herniation. The treatment is performed with intravenous antibiotic therapy and mastoidectomy. The most frequent sequel is visual loss due to optic atrophy and nerve compression.

\section{Discussion and results}

Otitis media are among the most prevalent diseases of childhood and represent the main reasons for pediatric consultations and the main cause of prescription of antibiotics for children. There is a great concern about this subject, since the indiscriminate use of antibiotics can determine resistant strains and a higher rate of complications. Usually, its course is indolent, but it can result in complications that usually require hospitalizations for intravenous therapy and sometimes surgical treatment. The most frequent otopathy in children is OMA, which is the inflammatory and infectious process of the middle ear mucosa, associated with the presence of secretion, characterized by rapid onset of the clinical picture and usually preceded by an upper airway infection (IVAS) ( In this paper, we present the results obtained in this paper. Usually, the resolution of the case is complete, but it can complicate and evolve into WTO. The main risk factors for the development of AOM are: IVAS, attending nurseries and nurseries, exposure to tobacco, allergies, low immunity, cleft palate, craniofacial anomaly, Down syndrome, other causes of auditory tube dysfunction, such as adenoid hypertrophy, Early interrupted breastfeeding, male sex, and low socioeconomic and cultural level.

Controlling such factors reduces the incidence of AOM, and its complications. Currently, the main risk factor for the development of complications is the delay in care by the public health system and the lack of public information regarding the subject, which results in a longer time of disease evolution and a potential risk of complication. The WTO usually results from a untreated suppurative AOM, and in rarer situations of unregenerate traumatic perforations and necrotizing otitis media. The rupture of intratympanic homeostasis will trigger a series of histological changes in the middle ear mucosa that would change in the direct ratio of future aggressions. Among the main forms of WTO are the non-cholesteatomatous OMC and OMCc, and cholesteatoma can be primary, secondary or congenital. Cholesteatoma is defined as an epidermoid cyst, with independent and progressive growth, with destruction of adjacent tissues, especially bone tissue. Because of this destructive potential, most of the complications are associated with OMCc.

The morbimortality of intra- and extracranial complications has drastically reduced in recent years, but serious cases have yet to be addressed. Extracranial is more common than intracranial, but these are more serious and lethal. Considered the most lethal complication of most articles, brain abscess deserves special attention. It evolved from extradural abscesses and subdural empyemas, which are less severe affections, so a high suspicion should be maintained regarding the formation of intracranial purulent collections. The brain abscess has 3 clinical stages that have been cited previously, and when treated correctly in the first stage, can evolve to complete cure without sequelae. The diagnostic difficulty is due to its non-specific signs and symptoms, such as evidence of intracranial hypertension and compressive signs. Therefore, all patients with suspected intracranial complications should undergo CT with contrast for an earlier diagnosis.

In brain abscess, antibiotics of choice should be cephalosporin of 3rd (ceftriaxone) or 4th generation (ceftazidime), associated with chloramphenicol, clindamycin, vancomycin, metronidazole or gentamicin. Such a scheme should also be used for other intracranial complications, $25 \%$ of patients with intracranial complications were treated with ceftriaxone alone; the other $75 \%$ required a therapeutic regimen with 2 or more antibiotics, one of which was ceftriaxone.

When the patient presents with cholesteatoma and evolves with more important sensorineural hypoacusis, tinnitus, otorrhea, vertigo, nystagmus and nausea and vomiting, a labyrinthic fistula should be suspected, since it is the most frequent complication related to OMCc. Subperiosteal abscess is the most common extracranial complication, and it occurs frequently in children. It is estimated that $4 \%$ of the AOM and $1 \%$ of the WTO develop with this type of complication. One should suspect this diagnosis when there is retroauricular bulging, anteroinferior displacement of the auricle and deletion of the retroauricular sulcus. The patient presents with fever, intense pain, toxemia and, sometimes, otorrhea. Four-generation intravenous antibiotic therapy should be used. Contrast CT is the gold standard examination when any complication is suspected in a patient with otitis media after a thorough medical history and physical examination. When the doctor knows the possible complications and their respective signs and symptoms, the diagnosis is early and the prognosis better. CT reveals 
bony details, abscesses, visualization of the sinuses (thrombus), etc., besides assisting in the surgical planning and evaluation of the extent of the disease. Its sensitivity ranges from $87.2 \%$ to $100 \%$.

Patients with culture and antibiogram positive for S. pneumoniae resistant, have more aggressive infections, increasing the chances of having suppurative complications and the necessity of intervention. The development of pneumococcal vaccines, targeting middle ear diseases, is a highly relevant issue. The serotype chosen was based on invasive pneumococcal diseases and their association with multidrug resistant organisms. The recent introduction of these vaccines is the future of research in this area. The treatment of complications in general is based on myringotomy with culture and antibiogram, intravenous antibiotic therapy as early as possible and mastoidectomy in all cases related to WTO or recurrent complications. Intracranial collections of all cases were drained together with mastoidectomy, but only $13 \%$ of brain abscesses, the remainder being performed in another surgical time.

\section{Conclusion}

Extracranial complications are more common, but intracranial complications are more lethal. WCO and WTO can progress to complications, but bacterial flora is different among them, which is important in the therapeutic orientation. OMCC is responsible for most of the complications, due to its Osteolytic and destructive. $\mathrm{CT}$ is mandatory when any otogenic complications are suspected. The extracranial complications are treated exclusively by otorhinolaryngologists, while the intracranial ones, in the majority, need a multidisciplinary team, with the cooperation of the neurosurgery. The advent of antibiotic therapy dramatically reduced the incidence of intra- and extracranial complications by otitis media, as well as their mortality rates, although this may mask the symptoms and delay the diagnosis. The doctor needs to know the otogenic complications as well as their signs and symptoms and to do a detailed physical examination to have a high index of suspicion. Early diagnosis and early treatment is of the utmost importance.

\section{Acknowledgments}

None.

\section{Conflicts of interest}

Author declares that there are no conflicts of interest.

\section{References}

1. Caldas Neto S, Melo Junior JF, Martins RHG, Costa SS. Treatise on Otorhinolaryngology and Cervical Facial Surgery. $2^{\text {nd }}$ edition. São Paulo: Editora Roca Ltda; 2011.p.61-83.

2. Dib GC, Garcia LBS, Penido NO. How to diagnose and treat acute otitis media: a review of the recent literature. Rev Bras Med. 2005(1):34-38.

3. Lima Neto CR, Mendes FA, Silva IV, et al. Complications of chronic otitis media. Rev Fac Ciênc Méd. 2008;3:21-24.

4. Miura MS, Krumennauer RC, Lubianca Neto JF. Intracranial complications of suppurative chronic otitis media in children. Rev Bras Otorrinolaringol. 2005;71(5):639-643.

5. Maranhão ASA, Andrade JSC, Godofredo VR, et al. Intratemporal complications of otitis media. Braz $J$ Otorhinolaryngol. 2013;79(2):141-149.
6. Wu JF, Jin $\mathrm{Z}$, Yang JM, et al. Extracranial and intracranial complications of otitis media: 22-year clinical experience and analysis. Acta Otolaryngol. 2012;132(3):261-265.

7. Martins GSQ, Pinna MH, Tsuji RK, et al. Description of 34 patients with complicated chronic cholesteatomatous otitis media. Int Otorrinolaringol. 2008;12(3):370-376.

8. Agrawal S, Husein M, MacRae D. Complications of Otitis Media: An Evolving State. J Otolaryngol. 2005;34 Suppl 1:S33-39.

9. Costa SS, Selaimen FA, Bergamaschi JAP, Costa LM. How to diagnose and treat acute otitis media. RBM. 2011;68:253.

10. Mustafa A, Heta A, Kastrati B, Dresha SH. Complications of chronic otitis media with cholesteatoma during a 10-year period in Kosovo. Eur Arch Otorhinolaringol. 2008;265(12):1477-1482.

11. Migirov L, Duvdevani S, Kronenberg J. Otogenic intracrania complications: A review of 28 cases. Acta Oto-Larymgologica. 2005;125(1):819-822.

12. Yorgancilar E, Yildirum M, Gun R, et al. Complications of chronic suppurative otitis media: a restrospective review. Eur Arch Otorhinolaryngol. 2013;270(1):69-76.

13. Penido NO, Iha LCN, Silva RN, et al. Labyrinthine fistula in chronic cholesteatomatous otitis media. Rev Bras Otorrinolaringol. 2003;4:521-525.

14. Shuman AG, Rizvi SS, Pirouet CW, et al. Hennebert's. Sign in Superior Semicircular Canal Dehiscence Syndrome: A Video Case Report. Laryngoscope. 2012;122(2):412-414

15. Dornelles C, Rosito LPS, Meurer L, Costa SS, et al. Correlation of ossicular chain in the intraoperative period with histological findings of cholesteatomas. Rev Bras Otorrinolaringol. 2007;73(6):738-743.

16. Marques F, Silva IV, Branco C, et al. Pediatric acute mastoiditis-risk factors for complications. Rev Ped Centr Hosp. 2013;22(1):13-18.

17. Bittencourt PAS, Gondim LMA, Ribeiro UJ, et al. Sigmoid and transverse sinus phlebitis and Bezold mastoiditis: case report and literature review. Rev Bras Otorrinolaringol. 2002;68(5):744-748.

18. Yonamine FK, Tuma J, Silva RFN, et al. Facial paralysis associated with acute otitis media. Braz J Otorhinolaryngol. 2009;75(2):228-230.

19. Santos AC, Ferreira CP, Silva KC, et al. Cawthorne and Cooksey exercises in elderly women: improvement of balance. Fisioter Mov. 2008;4:129-136.

20. Schiavetto RR, Haber DM, Cancian LR, et al. Gradenigo syndrome as the first manifestation of rhabdomyosarcoma. Int Arch Otorhinolaryngol. 2009;13(3):326-330.

21. Costa SS, Cruz OLM, Oliveira JAA. Otorhinolaryngology principles and practices. $2^{\text {nd }}$ edition. Porto Alegre: ARTMED EDITORIA S.A; 2009. p.254-282.

22. O'Connor TE, Perry CF, Lannigan FJ. Complications of otitis media in Indigenous and non-indigenous children. Med J Aust. 2009;191(9):S60-S64.

23. Oyamada LH, TMG Warrior, Nakaoka VY, et al. Acute otitis media. Braz J Surg Clin Res. 2014;6:134-139.

24. Penido NO, Toledo RN, Silveira PAL, et al. Thrombosis of the sigmoid sinus to chronic otitis media. Braz J Otorhinolaryngol. 2007;73(2):165-170. 